\title{
Estimation et gestion du risque de cancer broncho-pulmonaire engendré par les produits de filiation du radon
}

\author{
F. ROMERIO*, A. DONATH**
}

(Reçu le 6 mai 1996, révisé le 16 mai 1997, accepté le 19 mai 1997)

RÉSUMÉ Le radon est un gaz radioactif dégagé par les sols et les matériaux contenant du radium. Une fois inhalé, il se dépose dans l'appareil respiratoire, où ses produits de filiation irradient les tissus, provoquant un risque de cancer. L'estimation de ce risque s'effectue à travers une approche épidémiologique ou dosimétrique. La première utilise les données sur la mortalité des mineurs ayant été exposés au radon. On extrapole ensuite les résultats à la population générale. Plus récemment, on a essayé d'estimer le risque directement à partir de la population générale. L'autre approche essaie de calculer le taux de conversion entre la concentration de radon dans l'atmosphère ambiante et la dose absorbée par l'appareil respiratoire. On estime le risque de cancer à travers un coefficient qui a été déduit des études sur les survivants de Hiroshima et Nagasaki. La gestion du risque comporte la définition de principes généraux et d'un ensemble de recommandations. Dans le passé, l'ICRP pensait qu'il ne fallait pas intervenir sur les risques provoqués par les irradiations naturelles. Depuis 1984, la commission a changé d'avis et a établi des limites d'acceptabilité en ce qui concerne les concentrations de radon dans les habitations. Il faut relever que les estimations du risque de cancer sont entachées de marges d'incertitude relativement élevées. Les * jugements d'expert » jouent un rôle important. Les « jugements de valeur » en revanche jouent un rôle fondamental en matière de gestion du risque. $\grave{A}$ l'évidence, il s'agit d'un problème ayant un caractère transdisciplinaire et qui soulève la question du lien entre science et société.

ABSTRACT Estimation and management of risk for lung cancer induced by radon progeny. Radon is a radioactive gas released from soils and materials containing radium. Once inhaled in the respiratory tract, its decay products irradiate the tissues and provoke a risk of cancer. The estimation of this risk is carried out through an epidemiologic or a dosimetric approach. The former uses data on the mortality of miners who were exposed to radon. Then the results are extrapolated to the general population. More recently, some studies tried to estimate the risk directly on the general population. The latter approach tries to compute the rate of conversion between the radon concentration in the indoor environment and the dose absorbed by the respiratory tract. The risk of cancer is estimated through a coefficient which has been inferred from studies on the Hiroshima and Nagasaki survivors. The risk management requires the definition of general principles and of a set of recommendations. In the past, the ICRP stated that risks provoked by natural sources of irradiation should not be taken into consideration. Since 1984, it has changed its mind and limits of acceptability

* Centre universitaire d'étude des problèmes de l'énergie, Université de Genève, 1211 Genève 4, Suisse. ** Division de médecine nucléaire, Hôpital cantonal universitaire de Genève, 1211 Genève 4, Suisse. 
concerning radon concentration in dwellings have been defined. We should stress that the cancer risk estimations are tainted by uncertainties. "Experts judgements" play an important part. On the other hand, "value judgements" are essential in the field of risk management. Obviously, it is a problem which has a transdisciplinary character and which rises the question of the relationship between science and society.

\section{Introduction ${ }^{(1)(2)}$}

Nous analysons deux problèmes : l'estimation du risque de cancer bronchopulmonaire engendré par les produits de filiation du radon, en essayant de mettre l'accent sur les marges d'incertitude qui lui sont associées, ainsi que la gestion du risque, en cherchant de mettre en évidence les principes sur la base desquels on a défini les normes de radioprotection dans ce domaine.

Deux éléments jouent un rôle fondamental. Les « jugements d'expert », car, comme l'a relevé l'ICRP, « Each of the steps necessary to evaluate overall uncertainty involves the exercise of judgement and is therefore open to debate » (1991, p. 136-137), et les « jugements de valeur », car comme l'a relevé Stannard, médecin qui a suivi le programme nucléaire américain depuis les années 50, «...the standard setting process... inevitably involves judgment, selection, and factors outside of biology and medicine $(1988$, p. 1485).

Il s'agit d'un sujet qui présente deux difficultés. D'une part, il exige une approche transdisciplinaire, car il relève de la biophysique des radiations, de la médecine, de l'épidémiologie, des sciences humaines. D'autre part, il soulève la question de l'interaction entre science et société : en effet, les normes de radioprotection dépendent à la fois des études biophysiques et épidémiologiques et des jugements de valeurs admis ; ces derniers reflètent la sensibilité manifestée par une société donnée à l'égard du risque naturel et technologique; enfin, les études scientifiques dépendent dans une plus ou moins grande mesure des jugements subjectifs des experts consultés.

Ce sujet mérite une analyse de type diachronique, car les modèles épidémiologiques et dosimétriques, ainsi que les normes sur le radon, sont le résultat d'une évolution qui ne va pas s'arrêter. Dans cette perspective, nous fournissons un certain nombre de points de repères qui permettent de situer le problème du radon dans un contexte historique ${ }^{(3)}$. Nous rappelons aussi quelques problèmes qui sont à l'ordre du jour des programmes de recherche (NAS-

(1) Nous remercions vivement le Fonds national Suisse de la recherche scientifique qui a rendu possible la réalisation de ce travail, ainsi que trois lecteurs anonymes qui nous ont adressé des remarques très pertinentes.

(2) La signification des sigles se trouve à l'annexe B.

(3) Nous rappellons que le lien entre cancer du poumon et inhalation de radon a été montré en 1924 par Ludewig et Lorenser, de l'Institut du radium de la Bergakademie de Freiberg. Une analyse historique du problème du radon est fournie par Stannard (1988, p. 115-194), et par Jacobi (in ICRP, 1993, p. 39-45). 
NRC, 1994, p. 1-7). Nous mettons toutefois l'accent sur l'état actuel de la question. Nous discutons les études épidémiologiques et dosimétriques qui permettent d'estimer le risque provoqué par le radon et ses produits de filiation, ainsi que les problèmes qui se posent au niveau de la gestion du risque. Nous examinons le risque provoqué par le radon et ses produits de filiation sans toutefois prendre en considération d'autres facteurs qui, comme le tabagisme ou certains polluants chimiques, sont (ou peuvent être) à l'origine du cancer broncho-pulmonaire.

\section{TABLEAU I}

Décroissance du radon et de ses produits de filiation.

\begin{tabular}{|c|c|c|c|c|c|c|c|c|c|c|}
\hline \multicolumn{3}{|c|}{ 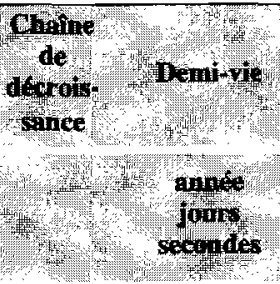 } & \multicolumn{2}{|c|}{$\begin{array}{l}\text { Eupreies } \\
\text { et forensites } \\
\text { or } \\
\text { Mev } \%\end{array}$} & \multicolumn{2}{|c|}{$\begin{array}{l}\text { Hortede } \\
\text { potentlelle } \\
\alpha\end{array}$} & \multicolumn{2}{|c|}{$\begin{array}{l}\text { Energes } \\
\text { ot linfensites } \\
\beta\end{array}$} & $\begin{array}{l}\text { Ene } \\
\text { Wht } \\
\text { Mev } \\
\text { Met } \\
\end{array}$ & 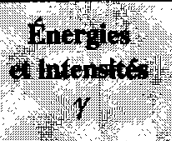 \\
\hline${ }^{238} \mathrm{U}$ & $4,5 \times 10^{9}$ & a & & & & & & & & \\
\hline${ }^{226} \mathrm{Ra}$ & 1600 & a & & & & & & & & \\
\hline${ }^{222} \mathrm{Rn}$ & 3,82 & j & 5,49 & 100 & 19,18 & $9,14 \times 10^{6}$ & - & - & 0,51 & 0,07 \\
\hline${ }^{218} \mathrm{Po}$ & 3,05 & $\mathrm{~m}$ & 6,00 & $\sim 100$ & 13,69 & 3616 & 0,33 & 0,02 & - & - \\
\hline${ }^{214} \mathrm{pb}$ & 26,8 & $\mathrm{~m}$ & - & - & 7,69 & 17860 & 0,65 & 50 & 0,29 & 19 \\
\hline & & & & & & & 0,71 & 40 & 0,35 & 36 \\
\hline & & & & & & & 0,98 & 6 & & \\
\hline${ }^{214} \mathrm{Bi}$ & 19,7 & $\mathrm{~m}$ & 5,45 & 0,012 & 7,69 & 13120 & 1,00 & 23 & 0,61 & 47 \\
\hline & & & 5,51 & 0,008 & & & 1,51 & 40 & 1,12 & 17 \\
\hline & & & & & & & 3,26 & 19 & 1,76 & 17 \\
\hline${ }^{214} \mathrm{Po}$ & 164 & $\mu \mathrm{s}$ & 7,69 & 100 & 7,69 & $1,82 \times 10^{-3}$ & - & - & 0,799 & 0,014 \\
\hline${ }^{210} \mathrm{~Pb}$ & 21 & a & & & & & & & & \\
\hline${ }^{216} \mathrm{~Pb}$ & stable & & & & & & & & & \\
\hline
\end{tabular}

$\lambda$ indique la constante de désintégration radioactive.

(Source: ICRP, 1981, p. 16-17).

\section{Quelques éléments de biophysique des radiations pour comprendre les effets du radon}

\section{I. Chaîne de décroissance du radon}

La chaîne de décroissance du radon 222 est illustrée par le tableau I. En particulier, on constate les faibles demi-vies des produits de filiation ; le rôle pré- 
pondérant joué par les émissions $\alpha$ (pour lesquels on indique aussi l'énergie potentielle $\left.{ }^{(4)}\right)$; deux radionucléides qui jouent un rôle très important : le ${ }^{218} \mathrm{Po}$ et le ${ }^{214} \mathrm{Po}$, qui émettent respectivement 6 et $7,69 \mathrm{MeV}$, et qui sont à l'origine de la quasi-totalité de la dose reçue par l'appareil respiratoire.

\subsection{Interactions entre radiations et matière}

Les particules expulsées de l'atome déposent l'énergie le long de leur trajectoire à travers la matière. Au cours de leur parcours, elles entrent en collision avec les électrons et ionisent ou excitent les atomes touchés. Au voisinage des ionisations primaires, le long de la trajectoire, se forment des grappes de matière ionisée. Le TLE (transfert linéaire d'énergie) exprime l'énergie cinétique déposée dans la matière par unité de parcours de la particule. Il est donné par l'expression suivante:

$$
\mathrm{TLE}=-\mathrm{d} E / \mathrm{d} s\left[\mathrm{keV} \mu \mathrm{m}^{-1}\right],
$$

où $E$ représente l'énergie déposée et $s$ la longueur de la trajectoire. Il faut souligner que la particule $\alpha$ possède un faible pouvoir de pénétration. Comme elle dissipe toute son énergie cinétique sur une distance relativement faible, la déposition d'énergie par unité de parcours est élevée. L'effet biologique est relativement important (Harley, Pasternack, 1972, p. 771-782). En revanche, les particules $\beta$ et $\gamma$ sont très pénétrantes, si bien que leur TLE est plus faible et leurs effets biologiques sont relativement moins dévastant.

\subsection{Effets biologiques}

Les radiations agissent sur la cellule de manière directe, en provoquant des lésions au niveau de l'ADN (acide désoxyribonucléique), ainsi que de façon indirecte, à travers la formation de radicaux libres (molécules avec un nombre impair d'électrons). L'ADN représente le constituant le plus radio sensible de la cellule. La rupture de ses brins peut conduire à la mort de la cellule ou provoquer des effets mutagènes ou carcinogènes. Il existe toutefois un système enzymatique qui dans une certaine mesure peut réparer ces lésions. Les ruptures peuvent être simples ou doubles (rupture d'un seul brin de l'hélice, respectivement des deux brins de manière simultanée). Les ruptures doubles sont beaucoup plus difficilement réparables. Dans le cas des radiations ayant un TLE élevé (notamment les radiations $\alpha$ ), une seule particule peut provoquer une double lésion de l'hélice de l'ADN. En revanche, dans le cas des radiations ayant un TLE faible, en général il faut deux événements pour provoquer ce type de lésion.

\subsection{Fonction dose-réponse}

Dans le domaine des radiations ionisantes, en général on utilise des fonctions de type linéaire-quadratique, c'est-à-dire :

(4) Pour les définitions et les unités de mesure, se rêférer à l'annexe. 


$$
F(D)=\left(a+b_{1} D+c_{1} D^{2}\right) \exp \left(-b_{2} D-c_{2} D^{2}\right) .
$$

$F(D)$ représente un indicateur des effets induits par les radiations (effets mutagènes, carcinogènes, mort des cellules); $D$ indique la dose reçue ; $a, b$ et $c$ sont des paramètres à estimer. L'exponentielle introduit un élément de correction, qui prend en considération l'atténuation des effets mutagènes et carcinogènes due à la mort des cellules. Le plus souvent, on pose $a=0$, car on admet que les mécanismes réparateurs peuvent être défaillants même à de très faibles doses. On a essayé de donner une interprétation biologique à $b_{1} D$ et $c_{1} D^{2}$. Le premier terme reflète les lésions irréversibles provoquées par une seule particule. Le deuxième terme reflète les lésions irréversibles engendrées par la combinaison de deux événements. Comme nous l'avons déjà relevé, dans le cas des radiations ayant un TLE élevé, un seul événement peut provoquer une lésion irréversible de la cellule. Le rapport $b_{1} / c_{1}$ est élevé et la fonction est de type quasi-linéaire. Au contraire, dans le cas des radiations ayant un TLE faible, en général il faut deux événements pour provoquer une lésion irréversible de l'hélice de l'ADN. Par conséquent, le rapport $b_{1} / c_{1}$ est relativement petit, et la fonction prend une forme parabolique. On pense que le fait de délivrer une dose de radiations à un débit de dose ${ }^{(5)}$ plus faible représente un facteur aggravant lorsque l'on a affaire à des radiations ayant un TLE élevé, et le contraire lorsque sont en question les radiations ayant un TLE faible. La figure 1 illustre différents cas de fonctions linéaires-quadratiques.

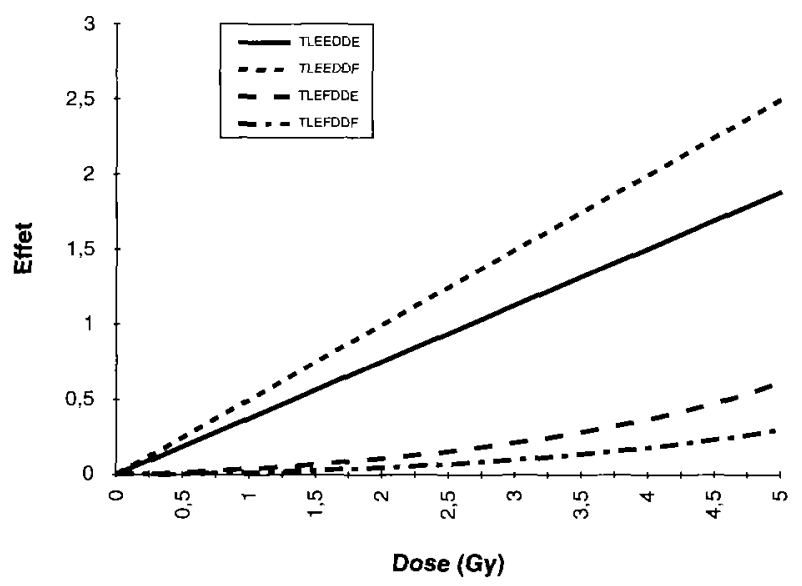

Fig. 1. - Fonctions linéaires-quadratiques. TLEEDDE : TLE élevé, débit de dose élevé; TLEEDDF : TLE élevé, débit de dose faible ; TLEFDDE : TLE faible, débit de dose élevé ; TLEFDDF : TLE faible, débit de dose faible.

(5) La dose s'exprime en Gy et le débit de dose en Gy $\mathrm{mn}^{-1}$. 


\subsection{Dose efficace}

La formule suivante définit l'équivalent de dose efficace $\left(H_{\mathrm{E}}\right)$ :

$$
H_{\mathrm{E}}=\Sigma_{\mathrm{R}} \Sigma_{\mathrm{T}} w_{\mathrm{R}} w_{\mathrm{T}} D_{\mathrm{R}, \mathrm{T}}[\mathrm{Sv} \text { ou rem }] .
$$

La dose reçue $\left(D_{\mathrm{R}, \mathrm{T}}\right)$ est multipliée à la fois par le « facteur de pondération pour les rayonnements $\left(w_{\mathrm{R}}\right)$ et par le «facteur de pondération pour les tissus $»\left(w_{\mathrm{T}}\right), w_{\mathrm{R}}$ permet de prendre en considération le fait que les différents types de radiations possèdent une plus grande probabilité de produire des effets biologiques irréversibles (dans le cas des radiations $\alpha, w_{\mathrm{R}}$ est égal à 20 ; dans le cas des radiations $\beta$ et $\gamma$, il est égal à 1 ). $w_{\mathrm{T}}$ permet de tenir compte du fait que les tissus et les organes du corps ne manifestent pas la même sensibilité aux radiations ionisantes (dans le cas de l'appareil respiratoire, $w_{\mathrm{T}}$ est égal à 0,12 ). On estime ce coefficient à partir des études épidémiologiques sur les survivants des explosions atomiques de Hiroshima et Nagasaki.

\section{Estimation du risque}

L'estimation du risque provoqué par les produits de filiation du radon s'effectue à travers une approche épidémiologique et une approche dosimétrique.

\subsection{L'approche épidémiologique}

L'approche épidémiologique cherche à estimer le risque engendré par les produits de filiation du radon sur la base de la mortalité enregistrée chez les mineurs ayant été exposés à ce type d'irradiation d'une part, ou chez la population vivant dans des régions où la concentration du radon dans les habitations est relativement élevée d'autre part. Les premières études épidémiologiques portant sur les populations de mineurs ont été effectuées à partir des années 30 en Europe et 50 aux Etats-Unis et Canada. Au cours des années 60, on a commencé à obtenir des résultats statistiquement significatives (Wagoner et al., 1964 ; Seltser, 1965 ; Archer et al., 1973 ; Cross et al., 1974 ; Sev et al., 1976). Les analyses épidémiologiques portant sur la population générale sont très récentes.

\subsubsection{Les études épidémiologiques sur les mineurs}

\subsubsection{Problèmes méthodologiques}

L'analyse épidémiologique soulève deux ordres de problèmes relatifs à la population des mineurs prise en considération, et à l'extrapolation des résultats obtenus à la population générale. Plus particulièrement, on peut citer les problèmes suivants :

- l'information : la fiabilité des estimations des doses d'irradiation subies par les mineurs et des diagnostics des causes de décès, 
- les facteurs perturbateurs : la distinction entre le risque engendré par le radon et le risque provoqué par d'autres facteurs (tabagisme et polluants présents dans la mine),

- le suivi des populations : les difficultés pratiques de suivre les mineurs jusqu'au décès,

- la taille de l'échantillon: de très grands échantillons sont nécessaires pour estimer des faibles risques,

- la sélection de la population: les mineurs ne sont pas complètement représentatifs de la population générale, car ils font partie d'un ensemble de classes d'âge déterminé et les variables physiologiques (comme le rythme respiratoire) sont influencées par les conditions de travail à l'intérieur de la mine,

- la pertinence des modèles : tout en s'adaptant aux données prises en considération, le modèle choisi peut ne pas refléter correctement le phénomène étudié et en particulier obtenir une validation de type biologique.

\subsubsection{Modélisation (NCRP, 1988)}

Ci-dessous, nous présentons quatre modèles permettant d'estimer le risque de cancer provoqué par les produits de filiation du radon. Ils ont été publiés par le NCRP en 1984, l'ICRP en 1987, le BEIR en 1988 et le GSF en 1992.

Il convient de rappeler que dans le domaine des radiations ionisantes on a défini deux types principaux de modèles :

- le modèle du risque absolu (ou modèle additif), qui suppose que les radiations provoquent un nombre supplémentaire de cancers qui est indépendant du nombre de cancers spontanés;

- le modèle du risque relatif (ou modèle multiplicatif), qui suppose que le nombre de cancers induits par l'irradiation est lié au nombre de cancers spontanés par le biais d'un multiple constant.

En général, on admet que l'utilisation du modèle du risque relatif a représenté un progrès par rapport au modèle du risque absolu. On souligne aussi le fait que ces deux types de modèles sont de moins en moins défendables en vue des données actuellement disponibles.

Le tableau II résume les principales caractéristiques des modèles cités, que nous présentons sous une forme qui facilite les comparaisons dans les encadrés qui suivent. On justifie l'hypothèse que le risque diminue au cours du temps par le fait que les cellules possèdent des mécanismes auto-réparateurs. Le facteur $k$ représente le rapport entre la dose absorbée par unité d'exposition par un individu à la maison et par un individu dans une mine. Il est calculé à travers des modèles dosimétriques. En général, on estime que la fraction d'atomes libres plus élevée, la dimension des aérosols plus petite 
et l'exposition continue, contribuent à accroître la dose absorbée par la population générale par rapport à la dose absorbée par les mineurs. En revanche, le rythme respiratoire moins élevé de la population générale contribue à la réduire. Il convient de signaler que la valeur proposée par BEIR IV $(k=1)$ a été révisée par un comité spécialement conçu en 1991, qui a proposé de la fixer à 0,7 pour les adultes et à 0,8 pour les enfants (NAS-NRC, 1991, p. 3).

\section{TABLEAU II}

Principales caractéristiques des modèles épidémiologiques examinés.

\begin{tabular}{|c|c|c|c|c|}
\hline 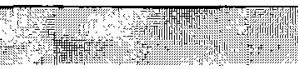 & $\operatorname{mep} 7$ & 4025 & 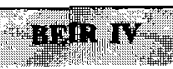 & (apd \\
\hline Fonction dose-tréponse & linéaire & linêaire & linéaire & linêaire \\
\hline $\begin{array}{l}\text { Modèle additif }(+) \\
\text { ou multiplicatif }\left({ }^{*}\right)\end{array}$ & + & * & * & * \\
\hline Période de latence (ans) & 5 & 10 & 5 & 4 \\
\hline $\begin{array}{l}\text { Influence de } l^{\text {'âge au }} \\
\text { moment de } l^{\prime} \text { 'irradiation } \\
\left(t_{0}\right)(\mathrm{R}=\text { risque })\end{array}$ & non & $\begin{array}{l}R \text { est } 3 \text { fois } \\
\text { supertieur si } \\
t_{0}<20 \text { ans }\end{array}$ & non & $\begin{array}{l}\mathrm{R} \text { dépend } \\
\text { de } 8 \text { classes } \\
\text { d'âge }\end{array}$ \\
\hline $\begin{array}{l}\text { Evolution du risque } \\
\text { depuis l'exposition }\end{array}$ & décroissant & constant & décroissant & décroissant \\
\hline $\begin{array}{l}\text { Influence du vieillisse- } \\
\text { ment ( } R=\text { risque })\end{array}$ & $\begin{array}{l}R \text { apparaît } \\
\text { aptès } 40 \text { ans }\end{array}$ & $\begin{array}{l}\mathbf{R} \text { reste } \\
\text { constant }\end{array}$ & $\begin{array}{l}R \text { diminue } \\
\text { aptès } 55 \text { ans }\end{array}$ & non \\
\hline Facteur $k$ & 1,4 & 0,8 & 1 & 1 \\
\hline
\end{tabular}

Sources : voir paragraphes suivants.

\section{Notations utilisées}

$H\left(t, t_{0}, D\right)$ Taux de mortalité due au cancer du poumon

$H_{0}(t) \quad$ Taux de mortalité spontanée due au cancer du poumon (indépendante de l'irradiation)

$t_{0} \quad$ Âge au moment de l'irradiation

$t \quad$ Âge au moment du décès

$D \quad$ Irradiation due aux produits de filiation du radon (en WLM) (on admet une seule irradiation à l'âge $t_{0}$ )

$P_{t_{0}} \quad$ Probabilité de survie jusqu'à l'âge $t_{0}$

$P_{t} \quad$ Probabilité de survie jusqu'à l'âge $t$ 


\section{NCRP 78 (NCRP, 1984, p. 151-161)}

$$
\begin{gathered}
H\left(t, t_{0}, D\right)=H_{0}(t)+\left(P_{t} / P_{0}\right) \times\left[\rho \times \phi\left(t-t_{0}\right) \times \mu(t) \times D\right] \\
\rho=10^{-5} \\
\phi\left(t-t_{0}\right)=0 \text { si }\left(t-t_{0}\right)<5 \\
=\exp \left[-\lambda\left(t-t_{0}\right)\right] \text { si }\left(t-t_{0}\right) \geq 5 \\
\text { où } \lambda=\ln 2 / 20 \\
\mu(t)=0 \text { si } t<40 \\
=1 \text { si } t \geq 40
\end{gathered}
$$

où $P_{t_{0}} / P_{t}$ représente un élément de correction, qui prend en considération les décès dus à d'autres causes.

Sources : mortalité des mineurs ayant travaillé dans des mines d'uranium, de zinc, de fer, de fluor, des ÉtatsUnis, Canada, Tchécosloyaquie, Suède et Terre Neuve.

ICRP $50($ ICRP, 1987, 30-38)

$$
\begin{gathered}
H\left(t, t_{0}, D\right)=H_{0}(t) \times\left[1+\sigma\left(t_{0}\right) \times \delta\left(t-t_{0}\right) \times D\right] \\
\sigma\left(t_{0}\right)=0,019 \text { si } t_{0} \leq 20 \\
=0,006 \text { si } t_{0}>20 \\
\delta\left(t-t_{0}\right)=0 \text { si }\left(t-t_{0}\right)<10 \\
=1 \text { si }\left(t-t_{0}\right) \geq 10
\end{gathered}
$$

Sources: mines d’uranium situées aux États-Unis, au Canada et en Tchécoslovaquie.

\section{BEIR IV (NAS-NCR, 1988, p. 38 et 77)}

$$
\begin{gathered}
H\left(t, t_{0}, D\right)=H_{0}(t) \times\left[1+\tau \times \eta\left(t-t_{0}\right) \times \gamma(t) \times D\right] \\
\tau=0,025 \\
\eta\left(t-t_{0}\right)=0 \mathrm{si}\left(t-t_{0}\right)<5 \\
=1 \text { si } 5 \leq\left(t-t_{0}\right)<15 \\
=0,5 \text { si }\left(t-t_{0}\right) \geq 15 \\
\gamma(t)=1,2 \text { si } t<55 \\
=1,0 \text { si } 55 \leq t<65 \\
=0,4 \text { si } t \geq 65
\end{gathered}
$$

Sources : les mines d'uranium de l'Ontario, du Saskatchewan, du Plateau du Colorado; une mine de fer en Suède. 
GSF (Jacobi, Henrichs, Barclay, 1993, p. 30-36)

$$
\begin{gathered}
H\left(t, t_{0}, D\right)=H_{0}(t) \times\left[1+\psi\left(t-t_{0}\right) \times \sigma\left(t_{0}\right) \times D\right] \\
\psi\left(t-t_{0}\right)=0 \text { si }\left(t-t_{0}\right) \leq 4 \\
=0,25 \times\left(t-t_{\mathrm{e}}-4\right) \text { si } 4<\left(t-t_{0}\right)<8 \\
=1 \text { si } 8 \leq\left(t-t_{0}\right) \leq 12 \\
\left.=\exp \left[-\lambda\left(t-t_{0}-12\right)\right] \text { si } t>12 \text { (où } \lambda=\ln 2 / 10\right) \\
\varpi\left(t_{0}\right)=0,036 \text { si } 20 \leq t_{0}<25 \\
=0,032 \text { si } 25 \leq t_{0}<30 \\
=0,030 \text { si } 30 \leq t_{0}<35 \\
=0,0285 \text { si } 35 \leq t_{0}<40 \\
=0,027 \text { si } 40 \leq t_{0}<45 \\
=0,0255 \text { si } 45 \leq t_{0}<50 \\
=0,0225 \text { si } 50 \leq t_{0}<55 \\
=0,018 \text { si } 55 \leq t_{0}<60
\end{gathered}
$$

Sources: les mines d'uranium de Saxe et Thuringe en Allemagne

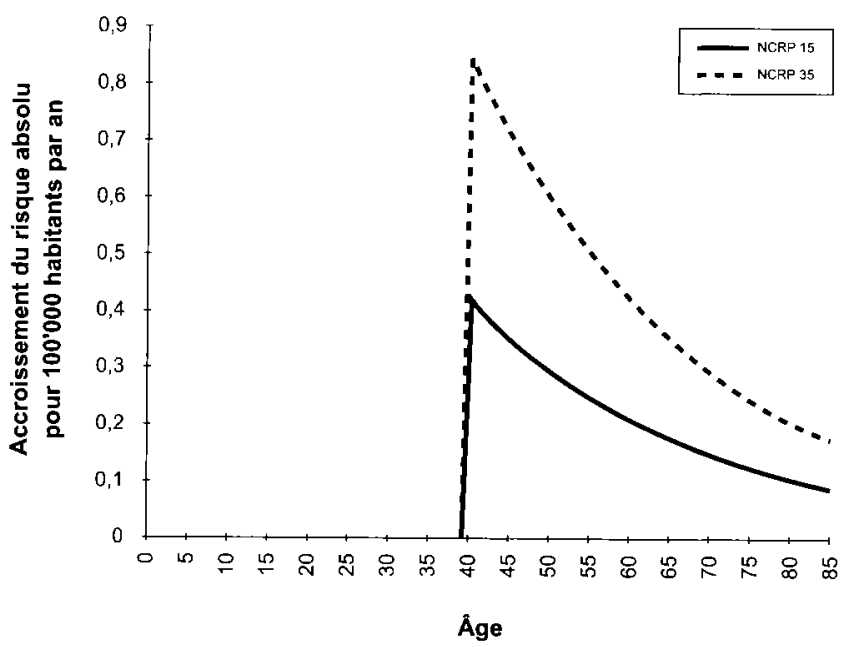

Fig. 2. - Accroissement du risque dû à l'exposition à 1 WLM à l'âge $t_{0}=15$ et $t_{0}=35$, modèle NCRP 78. 


\subsubsection{Quelques résultats}

La figure 2 montre l'accroissement du risque dû à l'exposition à 1 WLM à l'âge $t_{0}=15$, respectivement $t_{\mathrm{o}}=35$, calculé à travers le modèle additif NCRP 78. Les figures 3 et 4 illustrent l' « excès de risque relatif » (« excess relative risk») estimé à travers les modèles multiplicatifs BEIR IV, ICRP 50 et GSF. L' « excès de risque relatif » est représenté par l'expression qui figure entre parenthèse carrée dans les formules ci-dessus, moins 1 . Le tableau III fournit ce même indicateur pour quelques âges, et en particulier permet d'effectuer une comparaison entre les différents modèles, y compris le modèle NCRP 78.

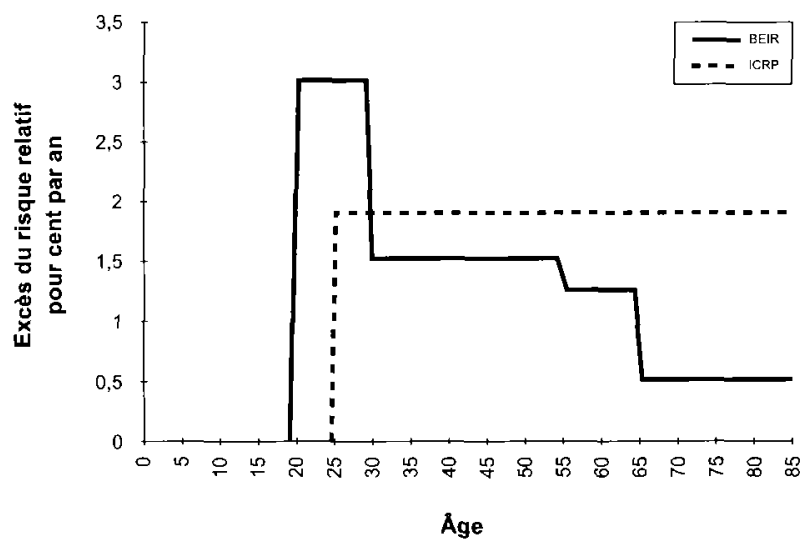

Fig. 3. - Excès de risque relatif dû à l'exposition à 1 WLM à l'âge $t_{0}=15$, modèles BEIR IV et ICRP 50.

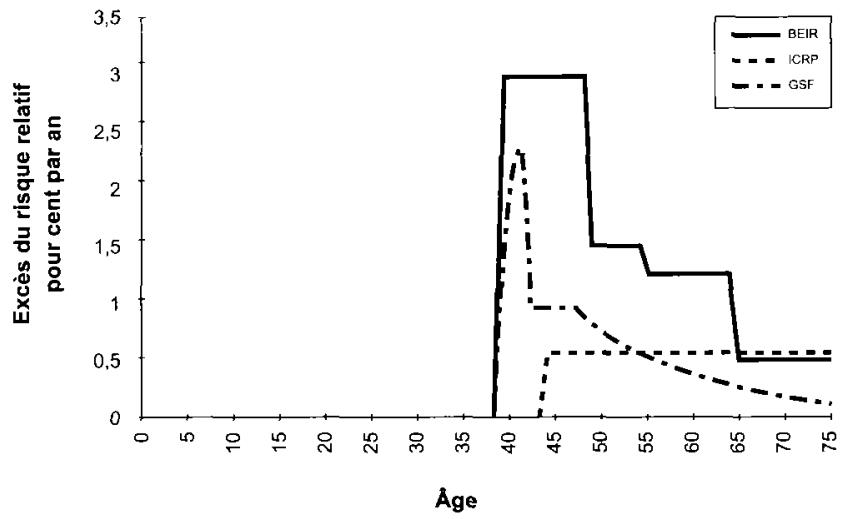

Fig. 4. - Excès de risque relatif dû à l'exposition à $I$ WLM à l'âge $t_{0}=35$, modèles BEIR IV,ICRP 50 et $\mathrm{GSF}$. 
TABLEAU III

Excès de risque relatif en pour cent par an dû à l'exposition à 1 WLM à l'âge $t_{0}$.

\begin{tabular}{|c|c|c|c|c|}
\hline Age & $\begin{array}{l}\text { CDP } 7 \\
\text { en } \%\end{array}$ & civs 6 & en & $\begin{array}{l}\text { GSF } \\
\text { on }\end{array}$ \\
\hline \multicolumn{5}{|c|}{ Exposition $t_{0}=15$ ans } \\
\hline 35 & 0 & 1,9 & 1,5 & - \\
\hline 50 & 0,5 & 1,9 & 1,5 & - \\
\hline 65 & 0,125 & 1,9 & 0,5 & - \\
\hline 85 & 0,06 & 1,9 & 0,5 & - \\
\hline \multicolumn{5}{|c|}{ Exposition $t_{0}=35$ ans } \\
\hline 50 & 1,0 & 0,6 & 1,5 & 0,8 \\
\hline 65 & 0,25 & 0,6 & 0,5 & 0,3 \\
\hline 85 & 0,125 & 0,6 & 0,5 & 0,05 \\
\hline
\end{tabular}

Sources: d'après les modèles ci-dessus; concernant le NRCP 78, cf. Samet, 1989, p. 754.

\subsubsection{Les études épidémiologiques sur la population générale}

\subsubsection{Problèmes méthodologiques}

Les études épidémiologiques sur la population générale comprennent les analyses «cas-témoins» et «écologiques ». Dans le premier cas, on compare deux groupes de personnes, souffrant et ne souffrant pas d'un cancer au poumon, par rapport aux expositions au radon subies dans le passé. En revanche, dans le deuxième cas, on essaie de vérifier s'il existe une corrélation entre l'apparition du cancer du poumon et la concentration moyenne de radon dans différentes régions géographiques. Lorsqu'on cherche à quantifier le risque encouru par la population générale, ces analyses ont l'avantage de fournir des résultats directs, qu'on ne doit pas extrapoler à partir des études sur les populations de mineurs. Elles soulèvent toutefois des problèmes méthodologiques et empiriques qu'il n'est pas toujours possible de surmonter. Dans le cas des études écologiques, on critique le fait de prendre en considération des groupes plutôt que des individus, et d'utiliser des indicateurs indirects de la concentration de radon dans les habitations (Stidley, Samet, 1993). On estime que tout au plus elles peuvent "générer des hypothèses de travail », qu'il faut toutefois tester à travers d'autres méthodes d'analyse. En revanche, les études cas-témoins fournissent des résultats plus fiables et sont en train d'ouvrir des pistes d'analyse prometteuses. Elles sont confrontées aux difficultés classiques de l'épidémiologie que nous avons évoquées au point 3.1.1.1 (Darby et Samet, 1994 ; Lubin et Samet, Weinberg, 1990 ; NAS-NRC, 1994, p. 69-71 ; Stidley et Samet, 1994). 


\subsubsection{Quelques résultats des études cas-témoins}

Trois études cas-témoins portant sur les pays Scandinaves et le New Jersey montrent que le risque de cancer broncho-pulmonaire augmente de manière significative avec l'augmentation de l'exposition au radon dans les habitations (Edling et al., 1984 ; Edling et al., Axelson, 1986 ; Schoenberg et al., 1990). Cette corrélation n'a pas pu être confirmée dans une étude très détaillée effectuée en Chine (Blot et al., 1990). D'autres analyses parviennent à des résultats assez contradictoires (Axelson, 1988 ; Lubin et al., 1994). Il faudra attendre encore quelques années avant de pouvoir tirer des conclusions plus précises de ces études. Pour l'instant, elles montrent que le problème de la détermination du risque provoqué par l'exposition au radon dans les bâtiments est toujours ouvert.

\subsection{L'approche dosimétrique}

L'approche dosimétrique essaie d'estimer le coefficient de conversion permettant de relier la concentration de radon dans l'atmosphère ambiante, à la dose absorbée par les cellules à risque de l'appareil respiratoire. Le calcul est effectué en prenant en considération un ensemble de facteurs de nature physique, physiologique et biologique, que nous examinerons ci-dessous. Le calcul de l'équivalent de dose efficace permet d'évaluer le risque provoqué par l'exposition aux produits de filiation du radon. À partir des études épidémiologiques effectuées sur les survivants des bombes atomiques de Hiroshima et Nagasaki, en effet on estime que le risque de développer un cancer mortel est de $5 \times 10^{-2}$ par $\mathrm{Sv}$. Les premières études dosimétriques ont été effectuées dans les années 50 et 60 (Bale, 1951 ; Harley, 1953 ; Bale et Shapiro, 1956 ; Chamberlain et Dyson, 1956 ; Altshuler et al., 1964 ; Jacobi, 1964 ; Parker, 1969). Elles ont créé les bases pour la construction des modèles publiés dans les années 80 , que nous présentons au point 3.2.2.

Il convient de relever que les études dosimétriques et de biophysique des radiations sont tributaires des expériences effectuées sur les animaux, car souvent il n'est pas possible d'obtenir les données nécessaires sur les êtres humains (NCRP, 1984, p. 114-144; NAS-NRC, 1990, p. 55).

\subsubsection{Les facteurs influençant la dose reçue par l'appareil respiratoire}

\section{Les facteurs physiques}

Ils comprennent le niveau de déséquilibre dans lequel se trouve le radon par rapport à ses produits de filiation, la fraction libre des produits de filiation et le diamètre des aérosols.

L'équilibre du radon par rapport à ses produits de filiation existe lorsque l'activité du premier est égal à l'activité de ces derniers. Il est rarement réalisé. Un facteur d'équilibre peu élevé entraîne une irradiation moindre de l'appareil respiratoire, car le dépôt des produits de filiation à vie courte est plus faible. 
La fraction libre des produits de filiation exerce une influence déterminante. Quelques pourcentages seulement d'énergie potentielle $\alpha$ sous forme d'atomes libres suffisent à augmenter de manière significative la dose reçue par unité d'exposition. Ceci est dû au fait que les atomes libres des produits de filiation se déposent rapidement par diffusion.

La dimension des aérosols a aussi une grande importance. Dans le cas de la dosimétrie du radon, on montre que la diminution de la dimension des particules a pour effet d'accroître la dose absorbée par les cellules à risque.

\section{Les facteurs physiologiques et biologiques}

Ils comprennent la morphométrie des bronches, le rythme respiratoire, le processus de déposition des particules, le transport ciliaire, l'épaisseur du mucus et de l'épithélium, et la localisation des cellules à risque.

Les deux modèles de l'appareil respiratoire les plus souvent utilisés dans ce domaine sont ceux de Weibel (1963) et de Yeh et Schum (1980), comprenant respectivement jusqu'à $8^{\prime} 388^{\prime} 608$ et $3 \times 10^{8}$ bronches dans la dernière génération ; à cet égard, il faut aussi mentionner le modèle mis au point par un groupe de travail de 1'ICRP en 1966 (Task Group on Lung Dynamics, 1966).

L'augmentation du rythme respiratoire a pour effet d'accroître la dose reçue par les cellules à risque de l'appareil respiratoire; la respiration nasale exerce aussi une certaine influence.

La déposition dans l'appareil respiratoire des atomes libres et des aérosols est calculée à travers les équations qui modélisent les processus de diffusion (laminaire, turbulente et pour une pause), de sédimentation et d'impaction inertielle.

À côté de la déposition des particules dans les générations de l'appareil respiratoire, il faut prendre en considération le transport ciliaire, qu'on peut chiffrer en cm par unités de temps.

Afin de calculer la dose absorbée par les cellules à risque, il est indispensable de connaître l'épaisseur du mucus et de l'épithélium. En outre, il faut modéliser la relation qui s'établit entre la source irradiante et la cible potentielle. Il est ainsi possible de déterminer l'énergie délivrée aux cellules à risque et l'énergie qui est dissipée dans l'air ou dans le mucus.

Les cellules à risques sont représentées par les cellules basales et les cellules sécrétoires, situées dans l'épithélium bronchique, dont la structure est illustrée par la figure 5 .

Il convient de rappeler que plusieurs études montrent qu'une proportion élevée des cancers diagnostiqués chez les mineurs ayant travaillé dans les mines d'uranium sont du type «petites cellules ». Le problème reste toutefois assez controversé (Gastineau et al., 1972, p. 857 ; NAS-NCR, 1988, p. 497-501; NAS-NRC, 1991, p. 186-188; Land et al., 1993, p. 234-243). 


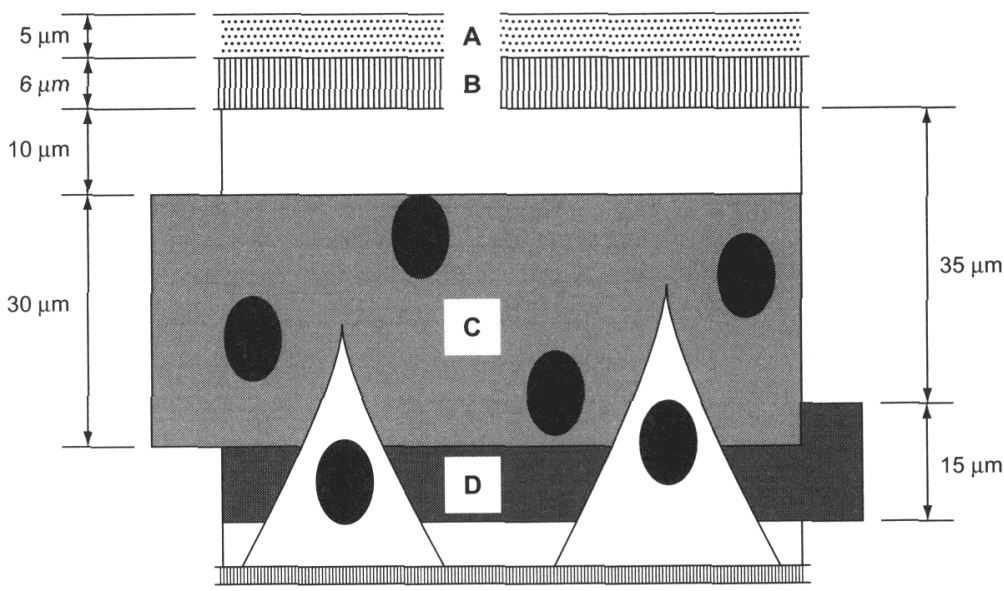

Fig. 5. - Structure de l'épithélium bronchique. A : Mucus ; B : Cil ; C : Cellules sécrétoires ; D: Cellules basales (d'après NAS-NRC, 1991, p. 197).

\subsubsection{Quelques résultats}

Le tableau IV montre quelques estimations du coefficient de conversion permettant de relier la concentration de radon dans l'atmosphère ambiante, à la dose absorbée par les cellules à risque de 1'appareil respiratoire. Il donne aussi les hypothèses concernant la fraction d'atomes libres et la dimension des particules qui sont à la base des calculs (Porstendörfer, 1994, p. 219-263). Le coefficient varie entre $5,5 \mathrm{mSv} / \mathrm{WLM}$ et $15.0 \mathrm{mSv} / \mathrm{WLM}$ (entre 0,06 et $0,17 \mathrm{mSv} / \mathrm{Bq} \mathrm{m}^{-3}$ ). Les hypothèses indiquées dans le tableau ne suffisent pas à expliquer ces différences. En fait, elles reflètent plutôt l'incertitude qui existe dans ce domaine. Il ne faut pas perdre de vue le fait que ces études représentent des synthèses d'un nombre impressionnant de recherches beaucoup plus ponctuelles. Pour une grande partie, elles utilisent donc les mêmes modèles et les mêmes données. Si ce n'était pas le cas, ces différences seraient nettement plus importantes. Il faut souligner que malheureusement quelquefois ces synthèses ne sont pas complètement transparentes, ne permettant pas ainsi de comprendre toutes les hypothèses qui ont été retenues dans les calculs. Le tableau indique aussi de combien il faut multiplier le coefficient calculé pour les adultes pour obtenir celui des enfants. On reviendra sur ce problème au point suivant. Il convient de relever que récemment 1'ICRP a proposé de prendre comme référence un coefficient de conversion de $4 \mathrm{mSv} / \mathrm{WLM}(0,045 \mathrm{mSv} /$ $\mathrm{Bq} \mathrm{\textrm {m } ^ { - 3 }}$ ) pour le public (1994a, p. 24). 


\section{TABLEAU IV}

Estimations du coefficient de conversion entre concentration de radon dans l'atmosphère ambiante et dose reçue par les cellules à risque de l'appareil respiratoire.

\begin{tabular}{|c|c|c|c|c|c|c|}
\hline (19) & 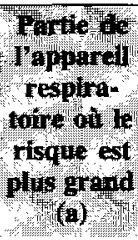 & $\begin{array}{l}\text { Fracton } \\
\text { Acitomes } \\
\text { Aibres en } \%\end{array}$ & 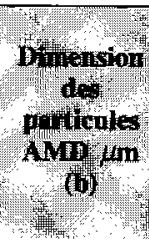 & 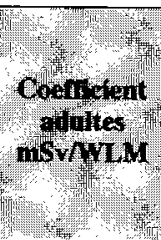 & 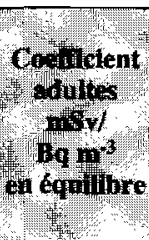 & 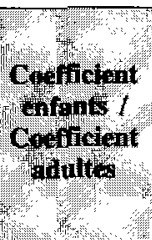 \\
\hline $\begin{array}{l}\text { Jacobi } \\
\text { et Eisfeld } \\
\text { (1980) }\end{array}$ & $\begin{array}{l}\text { Région } \\
\text { bronchique }\end{array}$ & $1,0-5,0$ & $0,1-0,5$ & 5,6 & - & $>1$ \\
\hline $\begin{array}{l}\text { Harley } \\
\text { et } \\
\text { Pasternack } \\
(1982)\end{array}$ & $\begin{array}{c}\text { Génération } \\
2 \text { et } 4\end{array}$ & 1,0 & 0,12 & 6,1 & 0,08 & 2,3 \\
\hline Nea $(1982)$ & $\begin{array}{c}\text { Région } \\
\text { bronchique }\end{array}$ & $1,5-2,5$ & $0,14-0,19$ & 5,5 & 0,06 & $\leq 2,0$ \\
\hline $\begin{array}{l}\text { NCRP } 78 \\
(1984)\end{array}$ & $\begin{array}{c}\text { Génération } \\
4\end{array}$ & 1,0 & 0,125 & 8,4 & 0,10 & 1,7 \\
\hline $\begin{array}{l}\text { ICRP } 50 \\
\text { (1987) }\end{array}$ & $\begin{array}{c}\text { Région } \\
\text { bronchique }\end{array}$ & 3,0 & 0,15 & 6,3 & 0,06 & 2,0 . \\
\hline $\begin{array}{l}\text { James } \\
(1988)\end{array}$ & $\begin{array}{c}\text { Région } \\
\text { bronchique }\end{array}$ & 5,0 & 0,1 & 15,0 & 0,17 & $\sim \mathrm{I}$ \\
\hline
\end{tabular}

(a) Les générations se réfèrent au modèle de Weibel.

(b) Activity median diameter

\subsection{Comparaison entre les deux approches}

Le tableau $\mathrm{V}$ permet de comparer un certain nombre de résultats obtenus à travers l'approche épidémiologique (études sur les mineurs) et l'approche dosimétrique. Le calcul du risque de mortalité dû à l'exposition chronique au radon à travers l'approche épidémiologique ne pose pas de problèmes particuliers. Pour estimer le même risque à travers l'approche dosimétrique, nous avons multiplié les coefficients du tableau IV par $\left(5 \times 10^{-2}\right)$, qui est le risque de développer un cancer mortel que l'ICRP attache à 1 Sv (ICRP, 1991, p. 133). On constate que les études dosimétriques parviennent à estimer un risque plus élevé que les analyses épidémiologiques. Il convient de relever que récemment l'ICRP a proposé de prendre comme référence un risque de $300 \times 10^{-6} \mathrm{WLM}^{-1}$ (1994a, p. 24). 
TABLEAU $\mathrm{V}$

Risque de mortalité par cancer du poumon dû à l'exposition chronique au radon, pour $10^{6}$ personnes par WLM.

\begin{tabular}{|lcccc|}
\hline & NEA & NCRP 78 & ICRP 50 & BETR IY \\
Epidémiologie & - & 130 & 230 & 350 \\
Dosimétrie & 275 & 420 & 315 & - \\
\hline
\end{tabular}

Source : voir texte et ICRP, 1991, p. 139.

L'influence de l'âge au moment de l'exposition a soulevé des controverses. Nous avons vu que les études dosimétriques indiquent que la dose reçue par WLM est entre 1 et 2 fois plus élevée chez un enfant qu'un adulte. Les études épidémiologiques font l'hypothèse que le risque est entre 1 et 3 fois plus élevé chez l'enfant. Les études les plus récentes semblent toutefois admettre que le risque n'est pas plus élevé pour un enfant que pour un adulte. Ceci grâce aux temps de latence relativement longs, qui permettent la réparation ou l'élimination des cellules endommagées, ainsi qu'au fait que le cancer du poumon est très rare chez l'enfant (ICRP, 1994a, p. 12 ; Xuan Xiang-Zehn et al., 1993).

Enfin, il convient de relever que ni les études épidémiologiques ni les études dosimétriques ne fournissent des informations précises sur l'influence exercée par des irradiations intermittentes ou délivrées à un débit de dose plus ou moins élevé. Le matériel réuni par BEIR VI semble toutefois confirmer que dans le cas du radon le risque de cancer augmente si le débit de dose de l'irradiation diminue (NAS-NRC, 1994, p. 34 ; cf. aussi Darby et Samet, 1994, p. 226).

\subsection{Discussion}

Nous avons essayé de mettre en lumière les incertitudes qui caractérisent les estimations du risque de cancer provoqué par les produits de filiation du radon. L'incertitude caractérise les modèles pris en considération, les hypothèses admises et évidemment les résultats obtenus. À chaque étape, les jugements plus ou moins subjectifs des experts jouent un rôle important. Land, du National Cancer Institute de Rockville, a très bien su interpréter cette situation en relevant que les modèles épidémiologiques (et, ajoutons-nous, dosimétriques) qu'on vient de présenter «provide a vivid illustration of the present level of uncertainty about the risks associated with indoor exposure to radon daughters, and of the extent to which committee choices and philosophies can influence the interpretation of essentially the same body of data " (NCRP, 1988, p. 123). Il faut reconnaître que jusqu'à récemment l'analyse de l'incertitude n'a pas été satisfaisante. On n'a pas effectué une analyse de sensibilité des variables prises en considération, ainsi que de leurs interactions. En particulier, on n'a pas entrepris une évaluation critique du rôle joué par les jugements d'expert dans ce domaine. Et pourtant BEIR V lui- 
même a reconnu que souvent les analyses statistiques doivent être complétées par des jugements d'expert, au point qu'au lieu de calculer des « intervalles de confiance », il faut évaluer des " intervalles de crédibilité » (NAS-NRC, 1990, p. 221).

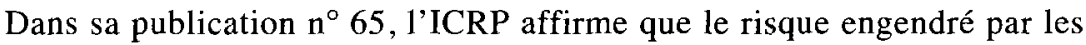
produits de filiation du radon doit être estimé à travers l'approche épidémiologique. En revanche, l'approche dosimétrique doit être utilisée pour « contrôler » les résultats obtenus par les études épidémiologiques (1994a, p. 8). Le point de vue de l'ICRP est légitime, car les estimations du risque de mortalité effectuées par les études dosimétriques se fondent sur les données des survivants des explosions atomiques de Hiroshima et Nagasaki. Or les caractéristiques de l'irradiation provoquée par ces explosions et de l'irradiation due à l'inhalation du radon sont très différentes. Dans le cas des bombes atomiques, la population a été irradiée par les rayons $\gamma$ et les neutrons ; l'irradiation a été de très courte durée, externe, uniformément répartie sur l'ensemble de l'appareil respiratoire. Au contraire, dans le cas du radon, l'irradiation est de type $\alpha$, de longue durée, interne, localisée (Land et al., 1993 ; Shigematsu et al., 1995). Les études épidémiologiques pourront bientôt profiter des travaux de Lubin et al., qui sont en cours d'évaluation, et qui portent sur 68000 mineurs et 2700 cancers (les analyses du BEIR IV avaient porté sur un échantillon nettement plus petit, comprenant 22190 mineurs et 360 cancers ; Lubin, 1994). Les études « cas-témoins » pourraient fournir des éléments nouveaux à l'évaluation du risque provoqué par le radon et ses produits de filiations. L'approche dosimétrique pourra bénéficier du nouveau modèle d'appareil respiratoire publié en 1994 par l'ICRP(6).

Dans l'avenir, il faut s'attendre à des progrès assez exceptionnels en biologie moléculaire. En particulier, il n'est pas impossible qu'on réussisse à mettre au point des méthodes d'analyse permettant de vérifier si un cancer a été provoqué par les radiations ionisantes. Récemment, des chercheurs ont identifié dans les cellules des tissus prélevés à des mineurs de l'uranium ayant développé une tumeur du poumon, des changements peu communs, ainsi que de petites délétions dans un gène inhibiteur de tumeurs (p53), qui peuvent indiquer des cancers générés par les produits de filiation du radon (Vähäkangas et al., 1992).

\section{Gestion du risque}

La gestion du risque radiologique comporte la définition de principes généraux et d'un ensemble de recommandations, voire de normes de radiopro-

(6) ICRP, 1994b. Un logiciel permettant d'effectuer des simulations est aussi disponible : cf. Dorrian et al., (1994). 
tection précises. Le problème du radon dans les habitations est devenu d'actualité dans les années 80 . En revanche, dans les mines, le problème se pose depuis plusieurs dizaines d'années (Cross et al., 1974 ; Stannard, 1988).

\subsection{Les principes}

Jusqu'à 1984, l'ICRP était de l'avis que les risques provoqués par l'irradiation naturelle ne devaient pas faire l'objet de recommandations spécifiques. Comme il y a des régions qui courent un risque de catastrophe naturelle plus élevé que d'autres, il était légitime, selon la Commission, d'admettre que certaines régions présentent un risque de développer un cancer radio-induit plus grand. Cette conception a été abandonnée depuis. Dans sa publication $n^{\circ} 39$, l'ICRP a relevé que les mesures de radioprotection sont souhaitables lorsqu'on est confronté à un niveau d'irradiation naturelle qui n'est pas négligeable pour la santé et qui peut être contrôlé (ICRP, 1984, p. 6).

Dès lors, il fallait prévoir des critères de décision. Comme les fonctions dose-réponse ne présentent pas de discontinuités, et du moment qu'on admet que le risque n'est pas nul même à de très faibles doses, se posait le problème de choisir, de manière plus ou moins éclairée, la limite d'acceptabilité du risque engendré par les produits de filiation du radon. De plus, comme les interventions peuvent être relativement coûteuses, il fallait prévoir un arbitrage entre le désir de diminuer le risque et le coût que l'on doit supporter. À cet égard, on pouvait s'inspirer d'un principe de radioprotection qui a été conçu pour les sources d'irradiation artificielles: «les expositions doivent être maintenues aux valeurs les plus faibles que l'on peut raisonnablement atteindre, compte-tenu des facteurs économiques et sociaux » (ICRP, 1989). Dans cet esprit, les pays qui ont introduit des normes sur le radon, ont prévu des interventions obligatoires lorsque la concentration de ce gaz à l'intérieur des habitations dépasse un certain seuil jugé inacceptable. En revanche, audessous de ce seuil, il appartient à l'individu de décider s'il souhaite effectuer des interventions en vue de réduire le risque (Bundesamt für Gesundheitswesen, 1992).

Pour fixer la limite d'acceptabilité, on a pris comme référence la concentration moyenne de radon dans l'air des habitations. On a aussi établi des comparaisons entre le risque provoqué par les produits de filiation du radon et d'autres types de risque, par exemple le risque de décéder d'un cancer du poumon pour un non-fumeur, ou le risque provoqué par les examens radiologiques en médecine (EPA, 1986, p. 10). Une procédure analogue a été adoptée dans le cas des sources d'irradiation artificielles, notamment dans le cas de l'énergie nucléaire, où on effectue une comparaison avec le risque provoqué par d'autres activités industrielles acceptées par le public. 


\subsection{Les recommandations}

Le tableau VI montre que les limites recommandées par la publication 65 de l'ICRP pour les habitations se situent entre 0,9 et $2,6 \mathrm{WLM}$ (200 et $600 \mathrm{~Bq} \mathrm{~m}^{-3}$, environ 4 respectivement 12 fois la concentration moyenne qu'on enregistre dans les habitations en Europe). Si elles sont dépassées, il faut prévoir l'assainissement du bâtiment. La dose efficace correspondante reçue par les cellules à risque est comprise entre 3 et $10 \mathrm{mSv}$ (l'ICRP suppose un coefficient de conversion de $4 \mathrm{mSv} \mathrm{WLM}^{-1}$ ). Il convient de rappeler que pour les sources d'irradiation artificielles, l'ICRP a fixé la limite de dose à $1 \mathrm{mSv}$ par an en moyenne pour les expositions prolongées. Pour les expositions de courte durée cette limite peut être portée à $5 \mathrm{mSv} \mathrm{a}^{-1}$, pourvu que la moyenne annuelle sur 5 ans ne dépasse pas $1 \mathrm{mSv}^{-1}$ (1991, p. 46). Le risque correspondant aux limites inférieures et supérieures des recommandations de 1'ICRP s'élève respectivement à $260 \times 10^{-6}$ et $790 \times 10^{-6}$ (on suppose un coefficient de conversion de $300 \times 10^{-6} \mathrm{WLM}^{-1}$ ). Il faut souligner que par rapport au passé les recommandations actuelles sont comprises dans une plus large fourchette. En effet, la limite recommandée par les publications 39 et 50 était de $250 \mathrm{~Bq} \mathrm{~m}^{-3}$.

\section{TABLEAU VI}

Limites recommandées pour les concentrations de radon dans les habitations, doses correspondantes et risques associés (a).

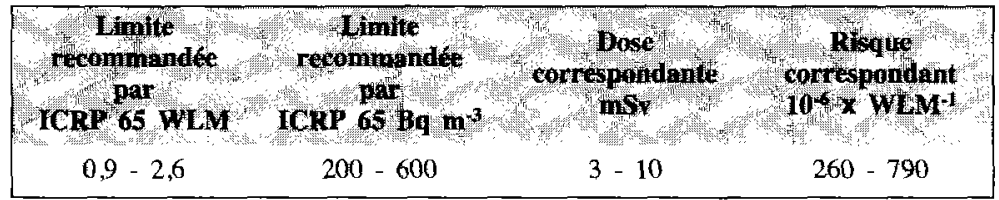

Source: ICRP, 1994a, tableau 5, p. 17 ; et 7 et 8, p. 23.

(a) On suppose que la fraction de temps passé à l'intérieur de l'habitation est de $80 \%$ (7000 heures) et le facteur d'équilibre de 0,4 .

\subsection{Discussion}

Les principes et les normes de radioprotection sont tributaires des études dosimétriques et épidémiologiques d'une part, et dépendent de l'attitude qu'une société manifeste envers les faibles risques d'autre part. Les « jugements de valeurs » jouent un rôle fondamental, car il n'est pas possible de prendre des décisions uniquement sur la base des résultats obtenus par les analyses scientifiques. Le changement d'attitude envers le risque naturel, qu'on a décidé de ne plus considérer comme une fatalité, nous semble très important. La fixation des limites d'acceptabilité sur la base d'une comparaison avec d'autres types de risque a soulevé des objections. Le problème met en effet en question des choix fondamentaux de société. À cet égard, il est assez significatif que les recommandations actuelles de l'ICRP 
soient comprises dans une fourchette relativement large, car elles doivent «s'adapter» aux principes de gestion du risque en vigueur dans les différents pays.

\section{Conclusions}

Nous avons essayé de montrer que le problème de l'estimation et de la gestion du risque de cancer provoqué par les produits de filiation du radon soulève la question de la relation existant entre science et société et exige une démarche transdisciplinaire. Nous avons aussi cherché à mettre en lumière l'importance des marges d'incertitude, ainsi que le rôle joué par les “jugements d'expert » et les «jugements de valeur ». Nous voudrions conclure en relevant l'intérêt d'approfondir l'étude de la relation entre science et société dans une optique transdisciplinaire. Cette étude est particulièrement nécessaire lorsque les problèmes sont très complexes, les incertitudes relativement élevées et les «jugements » incontournables, comme dans le cas du radon.

Plus particulièrement, il faut relever que dans le passé, l'étude du risque provoqué par les produits de filiation du radon a demandé un effort transdisciplinaire assez exceptionnel. Les résultats, malgré les approximations et les limites, sont remarquables. Nous pensons que dans l'avenir cette orientation devra être renforcée. La biologie moléculaire et l'histologie pourront fournir des informations extrêmement importantes à la dosimétrie. L'épidémiologie devra vérifier ses modèles pas seulement du point de vue statistique, mais aussi biologique. En outre, elle devra perfectionner ses méthodes d'analyse, notamment en perfectionnant les études «castémoins ». L'interaction entre l'épidémiologie et la dosimétrie restera fondamentale. L'effort le plus important devra toutefois être réalisé au niveau de la collaboration entre la médecine, les sciences exactes et les sciences humaines.

\section{Annexe A. Quelques définitions et unités de mesure utilisées dans les études sur le radon}

L'énergie potentielle $\alpha\left(\varepsilon_{\mathrm{p}}\right)$ d'un atome dans la chaîne de décroissance du radon représente le total de l'énergie $\alpha$ émise au cours de la désintégration de cet atome jusqu'à l'élément stable. Il s'exprime en $\mathrm{MeV}$ ou en $\mathrm{MeV} / \mathrm{Bq}$.

La concentration d'énergie potentielle $\alpha\left(c_{\mathrm{p}}\right)$ est donnée par l'énergie potentielle $\alpha$ de l'ensemble des produits de filiation du radon présents par unité de volume d'air. Elle s'exprime en $\mathrm{J} / \mathrm{m}^{3}$.

La concentration équivalente à l'équilibre $\left(c_{\mathrm{eq}}\right) \mathrm{d}$ 'un mélange de produits de filiation en déséquilibre est donnée par la concentration d'activité du radon, 
en équilibre radioactif avec ses produits de filiation, qui a la même concentration d'énergie potentielle $c_{p}$ que le mélange en question. Elle s'exprime en $\mathrm{J} / \mathrm{m}^{3}$.

Le facteur d'équilibre radioactif $(F)$ représente le rapport entre l'activité du radon dans l'air et l'activité de l'ensemble des descendants.

Le working level (WL) représente la concentration d'énergie potentielle $\alpha\left(c_{\mathrm{p}}\right)$ des produits de filiation du radon qui sont en équilibre radioactif avec une concentration d'activité du radon de $3700 \mathrm{~Bq} \mathrm{~m}^{-3}$.

Un working level month (WLM) correspond à l'exposition à 1 WL pendant 1 mois (160 ou 170 heures selon les études).

$\mathrm{Si}$ on indique avec $E_{\mathrm{p}}$ l'exposition à l'énergie potentielle $\alpha$ au cours d'une année, exprimée en WLM/a, et avec $C_{\mathrm{Rn}}$ la concentration de radon, exprimée en $\mathrm{Bq} \mathrm{m}^{-3}$, on $\mathrm{a}$ :

$$
E_{\mathrm{p}}=\left(8760 \times n \times F \times C_{\mathrm{Rn}}\right) /(160 \text { ou } 170 \times 3700)
$$

où $n$ représente la fraction de temps passée à l'intérieur de l'habitation et $F$ le facteur d'équilibre.

\section{Annexe B. Abréviations}

BEIR Committee on the biological effects of ionizing radiations.

EPA Environmental protection agency.

ICRP International commission on radiological protection.

NAS-NCR National academy of science - National research council.

NCRP National council on radiation protection.

NEA Nuclear energy agency.

UNSCEAR United nations scientific committee on the effects of atomic radiation.

\section{RÉFÉRENCES}

Altshuler N., Nelson N., Kuschner M. (1964) Estimation of lung tissue dose from the inhalation of radon daughters. Health Phys., 10, I 137-1161.

Archer V.E., Wagoner J.K., Lundin F.E. (1973) Lung cancer among uranium miners in the United States. Health Phys., 25, 351-371.

Axelson O. et al. (1988) Indoor radon exposure and active and passive smoking in : relation to the occurrence of lung cancer. Scand. J. Work Environ. Health, 14, 286-292. 
Bale W.F. (1980) Memorandum to the files, March 14, 1951: Hazards associated with radon and thoron. Health Phys. (Health Physics 25th Anniversary Issue) 38, $1062-1066$.

Bale W.F., Shapiro J.V. (1956) Radiation dosage to lung from radon and its daughter products, In : Proceedings of the first International Conference on the Peaceful Uses of Atomic Energy, New York, United Nations, 1956, v. 13, pp. 233-236.

Blot W.J. et al. (1990) Indoor radon and lung cancer in China. JNCI, 82, 1025-1030.

Bundesamt Fuer Gesundheitswesen (1992) Radonprogramm Schweiz "Rapros", Bern, März.

Chamberlain A.C., Dyson E.D. (1956) The dose to the trachea and bronchi from the decay products of radon and thoron. Brit. J. Radiol, 29, 317-325.

Cross F.T. et al. (1974) Evaluation of methods for setting occupational health standards for uranium miners, A research report for the National Institute for Occupational Safery and Health, Richland, Washington, Pacific Northwest Laboratory, 315 p.

Darby S.C., Samet J.M. (1994) Radon, In : Epidemiology of lung cancer (Samet J. Ed.) Marcel Dekker Publ, New York.

Dorrian M.-D. et al. (1994) LUDEP : past, present and future. Radiol. Prot. Bull., 159, Nov.

Edling C.H., Kling H., Axelson O. (1984) Radon in homes - a possible cause of lung cancer. Scand. J. Work Environ. Health, 10, 25-34.

Edling C.H., Wingren G., Axelson O. (1986) Quantification of the lung cancer risk from radon daughter exposure in dwellings - an epidemiological approach. Environ. Int., 12, 55-60.

EPA (1986) A citizen's guide to radon, Washington DC, US Department of Health and Human Services.

Gastineau R.M., Walsh P.J., Underwood N. (1972) Thickness of bronchial epithelium with relation to exposure to radon. Health Phys., 23, 857-860.

Harley J.H. (1953) Sampling and measurement of airborne daughter products of radon. Nucleonics, 11(7), 12-15.

Harley N.H., B.S. Pasternack (1972) Alpha absorption measurements applied to lung dose from radon daughters, Health Phys., 23, 771-782.

Harley N.H., B.S. Pasternack (1982) Environmental radon daughter alpha factors in five-lobed human lung. Health Phys., 42, 789-799.

ICRP (1981) Limits for inhalation of radon daughters by workers, Publication 32, Ann. ICRP. $6,1$.

ICRP (1984) Principles for limiting exposure of the public to natural sources of radiation, Publication 3. Ann. ICRP, 14, 1 .

ICRP (1987) Lung cancer risk from indoor exposures to radon daughters, Publication 50. Ann. ICRP, 17, 1 .

ICRP (1989) Optimization and decision-making in radiological protection, Publication 55. Ann. ICRP, 20, 1 .

ICRP (1991) 1990 Recommendations, Publication 60. Ann. ICRP, 21,1-3.

ICRP (1994a) Protection against radon-222 at home and at work, Publication 65. Ann. ICRP, $23,2$.

ICRP (1994b) Human respiratory tract model for radiological protection, Publication 66. Ann. ICRP, 24, 1-3.

Jacobi W. (1964) The dose to the human respiratory tract by inhalation of short-lived $222 \mathrm{Rn}$ - and 220Rn-decay products. Health Phys., 10, 1163-1174.

Jacobi W., Eisfeld K. (1980) Dose to tissues and effective dose equivalent by inhalation of radon222, radon-220 and their short-lived daughters, Gesellschaft für Strahlen- und Umweltforschung $\mathrm{mbH}$, München, $131 \mathrm{p}$. 
Jacobi W., Henrichs K., Barclay D. (1993) Verursachungs- Wahrscheinlichkeit von Lungenkrebs durch die berufliche Strahlenexposition von Uran-Bergarbeiten der WISMUT AG, Oberschleissheim, Institut für Strahlenschutz, Forschungszentrum für Umwelt und Gesundheit.

James A.C. (1988) Lung dosimetry, in : Radon and its decay products in indoor air (W.W. Nazaroff, A.V. Nero Eds.) Wiley, New York, pp. 259-309.

Jammet H., Pradel J. (1956) The problem of radon in uranium mines, in : Proceedings of the first International Conference on the Peaceful Uses of Atomic Energy, New York, United Nations, v. 13, pp. 219-221.

Land C.E. et al. (1993) Radiation-associated lung cancer : A comparison of the histology of lung cancers in uranium miners and survivors of the atomic bombings of Hiroshima and Nagasaki, Radiat. Res., 134, 232-243.

Lubin J.H., et al. (1994) Radon and lung cancer risk: A joint analysis of 11 underground miners studies, Bethesda National Institutes of Health.

Lubin J.H. et al. (1994) Radon exposure in residences and lung cancer among women : combined analysis of three studies, Cancer Causes Control, 5, 114-128.

Lubin J.H., Samet J.M., Weinberg C., (1990) Design issues in epidemiologic studies of indoor exposure to $\mathrm{Rn}$ and risk of cancer. Health Phys., 59, 807-817.

Ludewig P., Lorenser S. (1924) Untersuchungen der Grubenluft in den Schneeberger Gruben auf den Gehalt an Radium-Emanation. Z. Physik, 22, S. 178-185.

NAS-NRC (1988) Health risks of radon and other internally deposited alpha emitters, BEIR IV Report, National Academy Press, Washington DC.

NAS-NRC (1990) Health effects of exposure to low levels of ionizing radiation, BEIR V Report, National Academy Press, Washington DC.

NAS-NRC (1991) Comparative dosimetry of radon in mines and homes, Panel on dosimetric assumptions affecting the application of radon risk estimates, National Academy of Science, Washington DC.

NAS-NRC (1994) Health effects of exposure to radon : Time for reassessment?, BEIR VI Committee, National Academy Press, Washington DC.

NCRP (1984) Evaluation of occupational and environmental exposures to radon and radon daughters in the United States, Report No. 78, Bethesda, Maryland.

NCRP (1988) Radon, In : Proceedings of the 24th annual meeting, held on March 30-3l.

Palmer H.E., Perkins R.W., Stuart B.O. (1964) The distribution and deposition of radon daughters attached to dust particles in the respiratory system of humans exposed to uranium mine atmospheres. Health Phys., 10, 1129-1135.

Parker H.M. (1969) The dilemma of lung dosimetry. Health Phys., 16, 553-561.

Pirchan A., Sikl H. (1932) Cancer of the lung in the miners of Jachymov, Report of cases observed in 1929-1930. Am. J. Cancer, 16, 681-722.

Porstendoerfer (1994) Properties and behaviour of radon and thoron and their decay products in the air. J. Aerosol Science, 25, 2, 219-263.

Samet J.M. (1989) Radon and lung cancer. J. National Cancer Inst., 10, 745-757.

Schoenberg J.B. et al. (1990) Case-control study of residential radon and lung cancer among New Jersy women. Cancer Res., 50, 6520-6524.

Seltser R. (1965) Lung cancer and uranium mining : A critique. Arch. Environ. Health, 10, 923-935.

Sevc J., Kunz E., Placek V. (1976) Lung cancer in uranium miners and long-term exposure to radon daughter products. Health Phys., 30, 433-437. 


\section{CANCER BRONCHO-PULMONAIRE LIÉ AU RADON}

Shigematsu I. et al. (1995) Effects of A-bomb radiation on the human body, Tokyo, Bunkodo Co, pp. 62-69.

Stannard J.N. (1988) Radioactivity and health, A history, Prepared for the US Department of energy, (R. W. Baalman Jr., Ed.) Pacific Northwest Laboratory, pp. 115-194.

Stather J.W. (1994) Radiation carcinogenesis, Past, present and future. In : Radiation protection on the threshold of the 21 st century, Proceedings of a Conference organised by the NEA, held in Paris, II-13 January 1993, p. 31.

Stidley C.A., Samet J.M. (1993) A review of ecologic studies of lung cancer and indoor radon. Health Phys., 65 (3), 234-251.

Stidley C.A., Samet J.M. (1994) Assessment of ecologic regression in the study of lung cancer and indoor radiation. Am. J. Epidemiol., 139, 312-322.

Task Group On Lung Dynamics (1966) Deposition and retention models for internal dosimetry of the human respiratory tract. Health Phys., 12.

Vähäkangas K.H. et al. (1992) Mutations of p53 and ras genes in radon-associated lung cancer from uranium miners. The Lancet, 339, 576-580.

Wagoner J.K. et al. (1964) Cancer mortality patterns among US uranium miners and millers, 1950 through 1962, J. Nat. Cancer Inst., 32, 787-801.

Weibel E.R. (1963) Morphometry of the human lung, Springer Verlag, Berlin.

Xuan X.-Z et al. (1993) A cohort study in Southern China of tin miners exposed to radon and radon decay products. Health Phys., 64, (2), 120-131.

Yeh H.-C., Schum G.M. (1980) Models of human lung airways and their application to inhaled particle deposition. Bull. Math. Biol., 42, (2), 461-480. 